\title{
COVID-19 vaccination in patients receiving dialysis
}

\author{
Benjamin Wilde, Johannes Korth (), Michael Jahn and Andreas Kribben (10 ${ }^{凶}$
}

Patients receiving dialysis are at high risk of contracting SARS-CoV-2 and developing severe COVID-19. Established SARS-CoV-2 vaccination schemes might lack efficacy in these patients and a personalized approach is therefore necessary. Importantly, given the enhanced infection risks associated with dialysis, current vaccines do not replace non-pharmacological measures to prevent infection.

mRNA vaccines induce vaccine-specific immune responses in most patients receiving dialysis
Department of Nephrology, University Hospital Essen, University of Duisburg-Essen, Essen, Germany.

${ }^{凶}$-mail: andreas.kribben@ uni-due.de

https://doi.org/10.1038/ s41581-021-00499-z
Chronic kidney disease (CKD) is an important independent risk factor for severe COVID-19 with a fatal outcome $^{1}$. Mortality among patients receiving dialysis who develop COVID-19 is high ${ }^{1}$, and CKD outcompetes other risk factors for severe disease, such as age, diabetes and cardiovascular disease. Patients treated with dialysis are also particularly susceptible to SARS-CoV-2 infection, especially those who attend dialysis centres or reside in nursing homes (BOX 1). Non-pharmacological interventions to reduce the risk of infection, such as a facial mask mandate, regular SARS-CoV-2 testing and isolation measures for patients with COVID-19, are efficacious but challenging to implement in clinical practice.

The licensing of novel vaccines against SARS-CoV-2 in early 2021 enabled global vaccine rollouts that have greatly reduced mortality risks, but inequities in vaccine distribution have precluded access to this life-saving intervention for many individuals in the Majority World. However, CKD is often associated with immunodeficiency and other existing vaccines, such as hepatitis B vaccines, have reduced efficacy in patients receiving dialysis. Therefore, data on vaccine efficacy and immunological responses to vaccination obtained from healthy cohorts have limited relevance to cohorts of patients receiving dialysis, and it remains unclear whether current SARS-CoV-2 vaccination approaches are suitable for these patients.

Given their high risk of infection and severe disease, patients receiving dialysis have been prioritized for SARS-CoV-2 vaccination. Numerous studies have investigated the efficacy of these vaccines in patients receiving dialysis, and many of them used serial ELISA measurements of circulating antibodies against the viral spike (S) protein as a surrogate for vaccine-induced protection ${ }^{2}$. In healthy individuals, titres of $S$ protein-targeting antibodies correlate with neutralization capacity demonstrated in functional assays ${ }^{2}$. However, vaccination efficacy with clinical end points has not been determined in large clinical studies of patients receiving dialysis.
In general, older age and immunodeficiency negatively affect immunological responses to vaccination, and patients who were non-responders to hepatitis B vaccination seem to develop low anti-S protein antibody titres. By contrast, patients who have recovered from COVID-19 seem to have higher anti-S protein titres ${ }^{3}$. Nonetheless, mRNA vaccines induce vaccine-specific immune responses in most patients receiving dialysis and non-responders are a minority. However, whether the immune response in these patients is diminished and less protective than that of matched healthy controls remains a matter of debate. A recent interim analysis of a large cohort study compared the induction of SARS-CoV-2-neutralizing antibodies in COVID-19 -naive patients treated with dialysis after two doses of either the mRNA-based BNT162b2 vaccine $(n=55)$ or the adenovirus-based AZD1222 vaccine $(n=53)$, and healthy control participants $(n=162)^{4}$. Interestingly, in patients receiving dialysis, the BNT162b2 vaccine induced higher titres of neutralizing antibodies against the wild-type virus and variants (for example, the Delta variant) than the AZD1222 vaccine 33 days after the second dose. Moreover, across all variants tested, antibody titres following BNT162b2 vaccination were similar between patients receiving dialysis and healthy controls, whereas vaccination with AZD1222 was less efficacious in patients receiving dialysis. Patients who received AZD1222 had significantly lower neutralizing antibody responses against variants such as the Delta variant compared with healthy controls ${ }^{4}$. The difference between the AZD1222 and BNT162b2 vaccines was less pronounced in patients with previous infection treated with dialysis.

Of note, the data available mostly reflect the early vaccination response, and the durability and persistence of vaccine-induced immunity in patients receiving dialysis has not been assessed in detail. In healthy individuals, despite a reduction in antibody levels, SARS-CoV-2-binding antibodies persisted in all participants 6 months after the second dose of the mRNA- 1273 
Box 1 Mitigation of infection and severe disease risk in patients treated with dialysis

Several risk factors contribute to the increased risk of SARS-CoV- 2 infection and severe COVID-19 in patients receiving dialysis:

- Older age

- Residence in a nursing home

- Immunodeficiency or immunosuppression

- In-centre dialysis (high number of personal contacts before, during and after dialysis, and/or use of small dialysis rooms shared with other patients)

- High incidence of SARS-CoV-2 infection

Strategies to mitigate these risk factors include:

- Frequent SARS-CoV-2 testing

- Non-pharmacological measures for infection prevention, including enhanced ventilation in dialysis units and the use of face masks

- Grouping strategies with coordinated patient arrival and departure times, and modified dialysis treatment schedules

Vaccination (of patients and dialysis staff) is crucial to protect patients receiving dialysis, but factors that affect responses to vaccination, such as age, history of COVID-19 or immunosuppression, must be considered. Measures to ensure optimal vaccine-based protection include:

- Post-vaccination measurement of SARS-CoV-2-specific lgG to inform personalized vaccination schedules

- Use of vaccine boosters in patients with low or waning lgG levels

CS in patients receiving dialysis ... several unique risk factors have implications for patient care vaccine $^{5}$. However, functional variant-specific activity was lost in some individuals. In another study, SARS-CoV-2-specific antibody titres were assessed 6 months after infection in patients who were convalescent and receiving dialysis ${ }^{6}$. In this cohort, $85 \%$ of patients with seroconversion after infection still harboured SARS-CoV-2-specific antibodies, but titres declined significantly over time. A recent preprint indicates that vaccine-specific humoral and cellular immunity wane 4 months after two vaccine doses in patients receiving dialysis; at this time-point, $17.1 \%$ of these patients had lost the vaccine-specific T cell or B cell response $\mathrm{T}^{7}$. Booster immunizations are thought to counteract the decline in vaccine-induced immunity and are well established in vaccination protocols against other types of infectious disease. Administering a single vaccine dose to individuals with a prior history of COVID-19, including patients receiving dialysis, induces a substantial rise in SARS-CoV-2-specific antibody titres ${ }^{3}$ and has become a common strategy for protection in these patients. Likewise, booster immunizations might induce a humoral immune response in patients who did not respond to a standard two-dose immunization. A third dose of mRNA vaccine elicited a detectable humoral response in $54.5 \%$ of non-responding patients receiving dialysis ${ }^{8}$. Whether booster immunizations are also efficacious in counteracting waning of vaccine-specific immunity over time in patients receiving dialysis is currently under investigation.

SARS-CoV-2 vaccination is efficacious in patients receiving dialysis, although several unique risk factors have implications for patient care (BOX 1). Vaccination schedules should be personalized and take into account the response to vaccination, its longevity and the extent of protection conferred. Most studies have evaluated mRNA vaccines in patients receiving dialysis, but fewer data are available on vector-based vaccines. The vaccination schedule should also depend on the clinical history of the individual. COVID-19-naive patients need to complete the standard vaccination schedule, whereas patients with a history of COVID-19 usually only need one vaccine dose to build up the same level or even more extensive immunity compared with COVID-19-naive patients who have received two vaccine doses ${ }^{9}$. The time period between vaccine doses may vary, and longer intervals might offer some advantages, although the optimal timing remains unclear. Clinical tests are now available to measure serum SARS-CoV-2-specific IgG, which can be used to evaluate patient responses to vaccination and should allow the longevity of those responses to be monitored. Thus, patients with no response or declining immunity (for example, patients with current or past immunosuppression) can be identified and considered for further measures such as booster vaccine doses. Patients receiving dialysis after vaccination can still be infected with SARS-CoV-2 and be infectious. Consequently, non-pharmacological measures such as facial mask mandates, physical distancing, regular testing and isolation measures must be maintained ${ }^{10}$.

1. Goffin, E. et al. COVID-19 related mortality in kidney transplant and hemodialysis patients: a comparative, prospective registry based study. Nephrol. Dial. Transplan. https://doi.org/10.1093/ndt/ gfab200 (2021).

2. Khoury, D. S. et al. Neutralizing antibody levels are highly predictive of immune protection from symptomatic SARS-CoV-2 infection. Nat. Med. 27, 1205-1211 (2021).

3. Chan, L., Fuca, N., Zeldis, E., Campbell, K. N. \& Shaikh, A. Antibody response to mRNA-1273 SARS-CoV-2 vaccine in hemodialysis patients with and without prior COVID-19. Clin. J. Am. Soc. Nephrol. 16, 1258-1260 (2021).

4. Carr, E. J. et al. Neutralising antibodies after COVID-19 vaccination in UK haemodialysis patients. Lancet 398, 1038-1041 (2021).

5. Pegu, A. et al. Durability of mRNA-1273 vaccine-induced antibodies against SARS-CoV-2 variants. Science 373, 1372-1377 (2021).

6. Clarke, C. L. et al. Longevity of SARS-CoV-2 immune responses in hemodialysis patients and protection against reinfection. Kidney Int. 99, 1470-1477 (2021).

7. Dulovic, A. et al. Diminishing immune responses against variants of concern in dialysis patients four months after SARS-CoV-2 mRNA vaccination. Preprint at medRxiv https://doi.org/10.1101/2021.08. 16.21262115 (2021)

8. Dekervel, M. et al. Humoral response to a third injection of BNT $162 \mathrm{~b} 2$ vaccine in patients on maintenance hemodialysis. Clin. Kidney J. https://doi.org/10.1093/ckj/sfab 152 (2021).

9. Flaxman, A. et al. Reactogenicity and immunogenicity after a late second dose or a third dose of ChAdOx $1 \mathrm{nCoV}-19$ in the UK: a substudy of two randomised controlled trials (COV001 and COV002). Lancet 398, 981-990 (2021).

10. Bergwerk, M. et al. Covid-19 breakthrough infections in vaccinated health care workers. N. Engl. J. Med. https://doi.org/10.1056/ NEJMoa2109072 (2021).

\section{Competing interests}

B.W. was funded by the Dr. Werner Jackstädt Foundation and received travel reimbursements and/or honoraria from Alexion, Chiesi, Hexal, Otsuka, TEVA, Sanofi and Wyeth. A.K. received travel reimbursements, honoria and/or grants from Ablynx, Advitos, Alexion, Amgen, Astellas, AstraZeneca, Baxter, Bayer Vital, Bristol Myers Squibb, B. Braun Avitum, Binding Site, Charité, Chiesi, Cyto Sorbents, Exthera Medical, GlaxoSmithKline, GE Healthcare, InflaRx, Jafron, Janssen-Cilag, Kyowa Kirin, Neovii Biotech, Novartis, Otsuka, Peripal, Pfizer, Reata Pharmaceuticals, Sanofi-Aventis, Shire-Takeda, Syneos Health and Vifor Fresenius. The other authors declare no competing interests. 